1

2

3

4 in the catabolism of the herbicide dicamba in Rhizorhabdus

\title{
dicambivorans Ndbn-20
}

$6 \mathrm{Na} \mathrm{Li}^{a}$, Qian Peng ${ }^{b}, \mathrm{Li} \mathrm{Yao}^{c}$, Qin $\mathrm{He}^{b}$, Jiguo Qiu ${ }^{b}$, Hui Cao ${ }^{b, *}, \mathrm{Jian}_{\mathrm{He}}^{b, *}$, Qiuhong

$7 \quad \mathrm{Niu}^{a}$, Yunfeng $\mathrm{Lu}^{a, d}$, Fengli Hui ${ }^{a}$

9 chlorogentisate in Rhizorhabdus dicambivorans Ndbn-20 

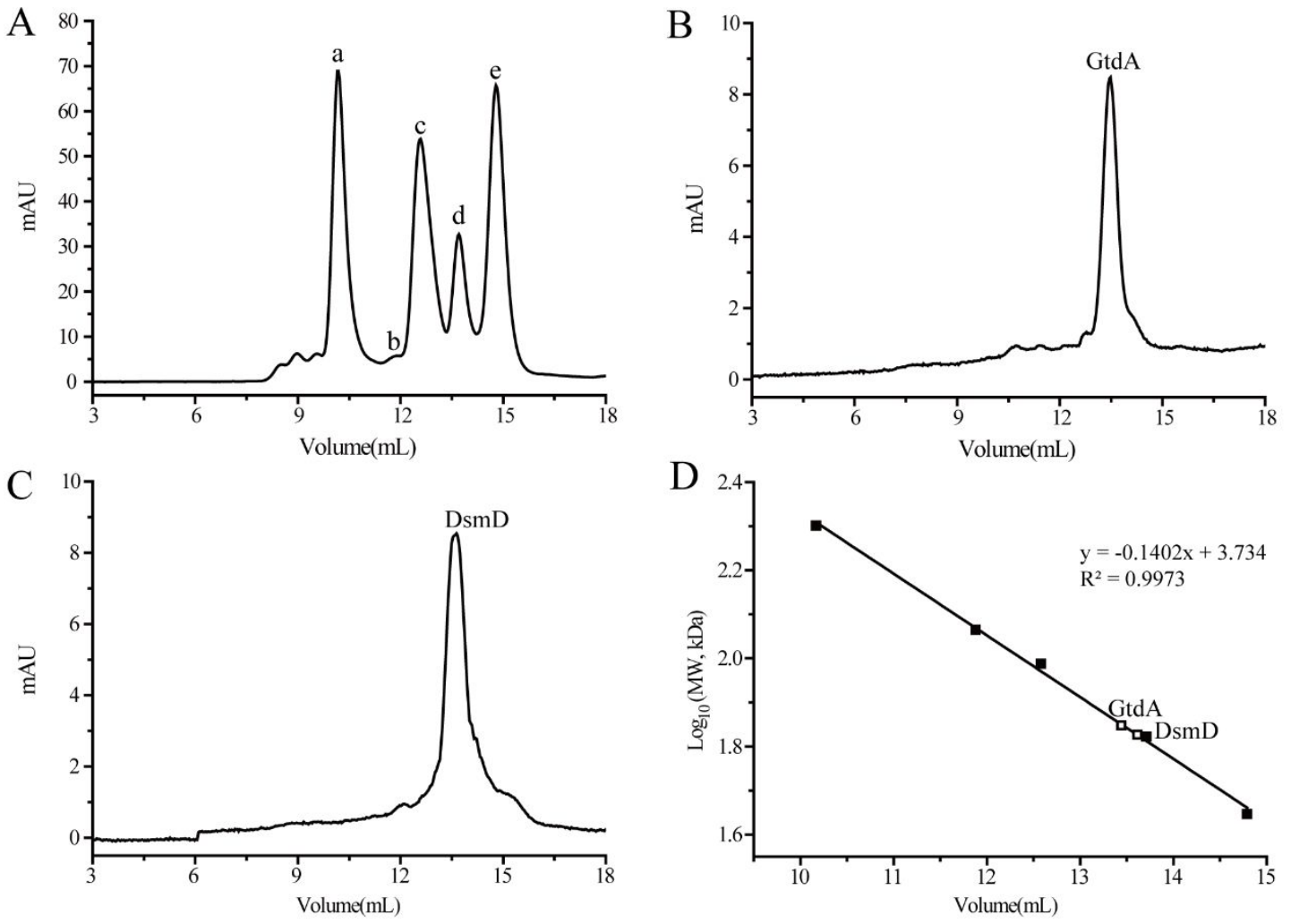

Figure S1. Gel filtration of the purified GtdA and DsmD. (A) The peak volumes of standard proteins: a, myosin (200 kDa, $10.17 \mathrm{ml})$; b, $\beta$-galactosidase (116.0 kDa, 11.88 $\mathrm{ml})$; c, phosphorylase b (97.2 kDa, $12.58 \mathrm{ml})$; d, bovine serum albumin (66.4 kDa, $13.71 \mathrm{ml})$; e, ovalbumin $(44.3 \mathrm{kDa}, 14.79 \mathrm{ml})$; (B) The peak volume of native GtdA (13.48 ml); (C) The peak volume of native DsmD (13.64 ml); (D) The calibration line of standard proteins and molecular mass determination of GtdA (70.0 kDa) and DsmD (66.4 kDa). 

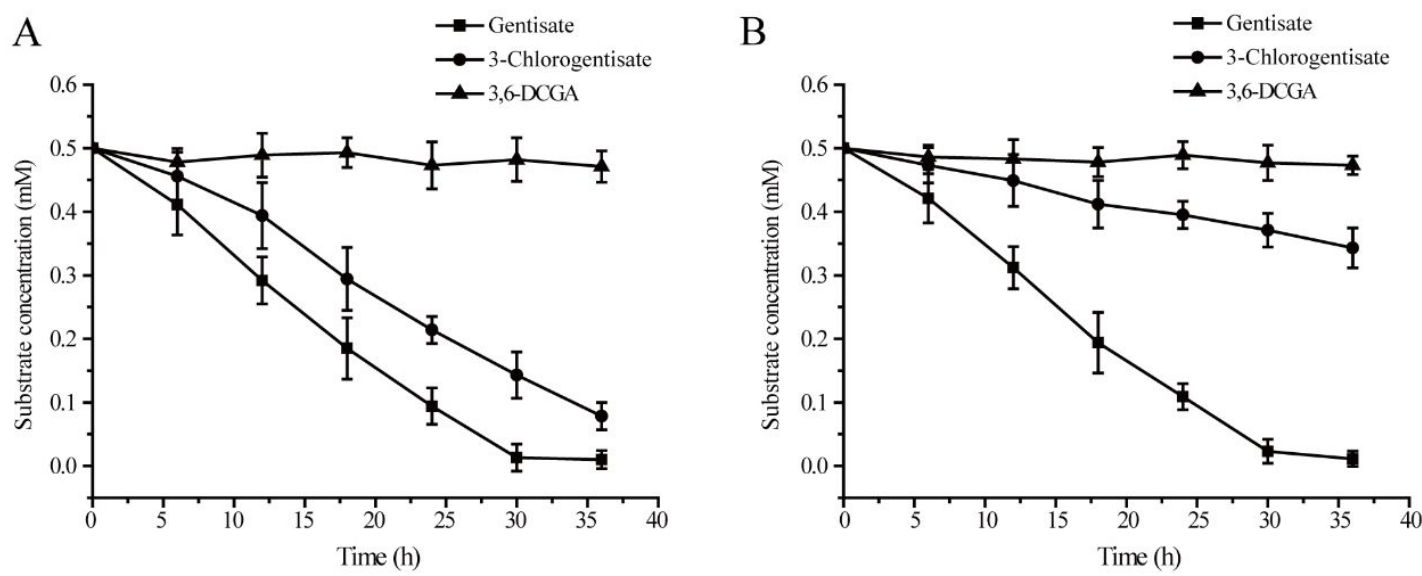

31

32

Figure S2. Time course of gentisate, 3-chlorogentisate and 3,6-DCGA degradation by the mutant Ndbn-20 $\Delta d s m H 2$ (A) and the recombinant DC-2(pBBR-gtdA) (B). The data were derived from three independent measurements, and the error bars indicate standard deviations.
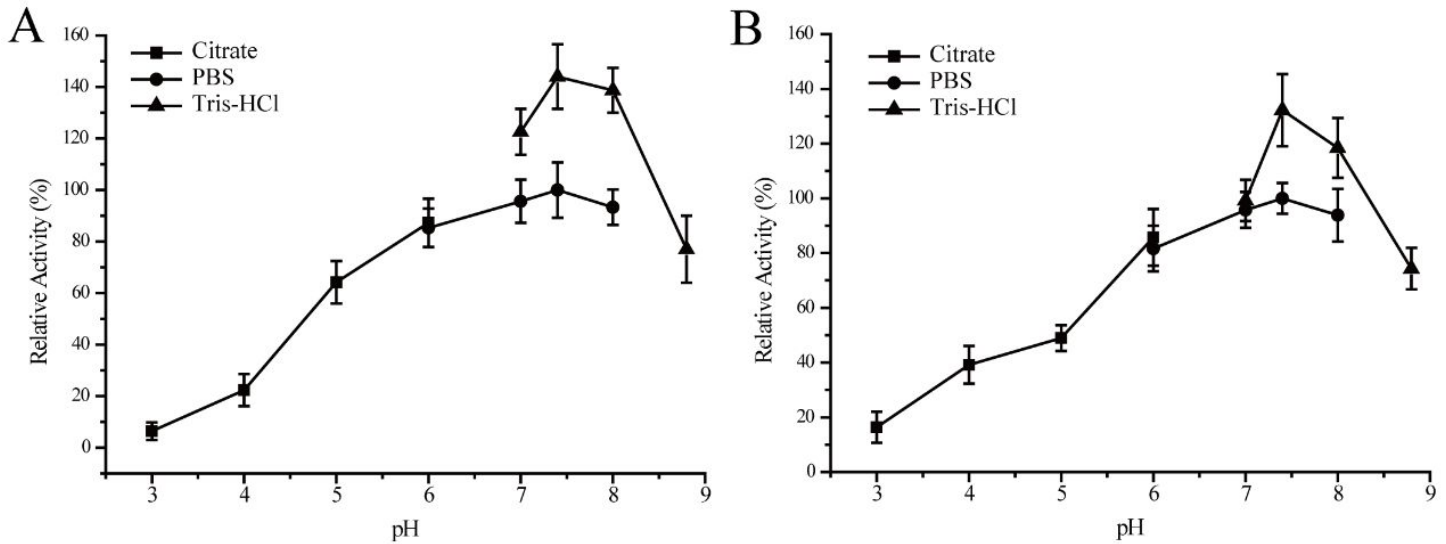

Figure S3. Effects of $\mathrm{pH}$ on the activities of GtdA (A) and DsmD (B). Three buffering systems $(50 \mathrm{mM}):$ citrate buffer ( $\mathrm{pH} 3.0$ to 6.0 ), PBS buffer (pH 6.0 to 8.0 ) and $\boldsymbol{\Delta}$ Tris- $\mathrm{HCl}$ buffer ( $\mathrm{pH} 7.0$ to 8.8 ). The relative activity was calculated by assuming that the activity at $\mathrm{pH} 7.4$ in PBS buffer was $100 \%$. 
43

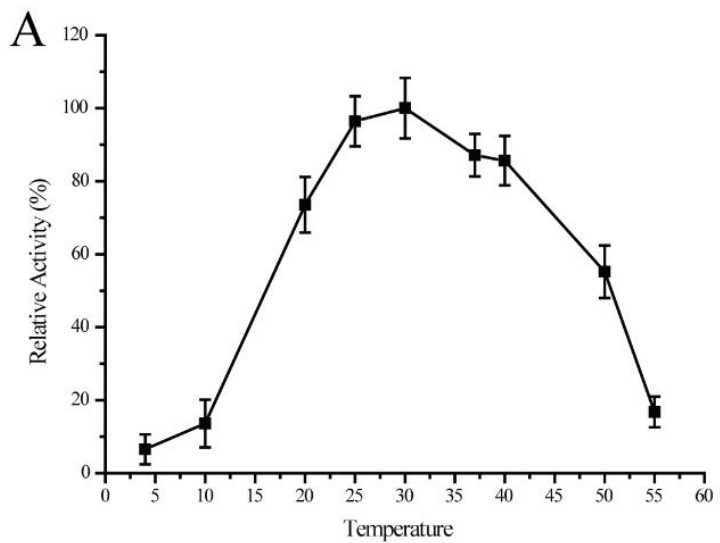

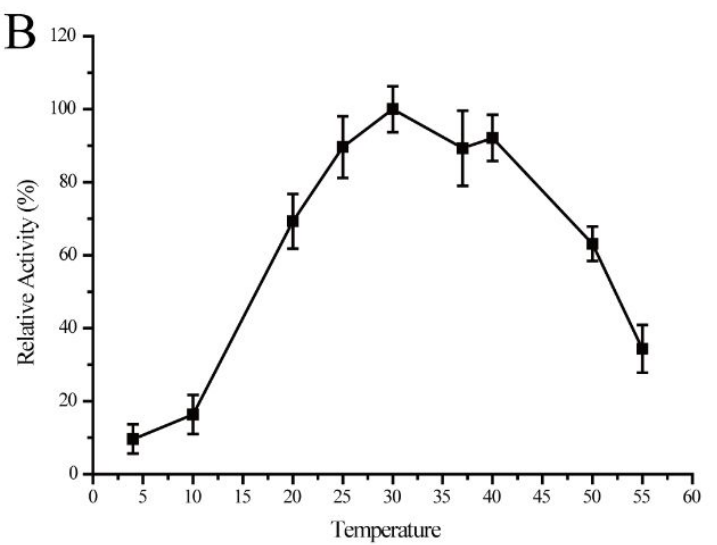

44

45

46

47 observed at $30^{\circ} \mathrm{C}$ was $100 \%$.

Figure S4. Effects of temperature on the activities of GtdA (A) and DsmD (B). GtdA and DsmD were incubated in $50 \mathrm{mM}$ PBS buffer ( $\mathrm{pH}$ 7.4) at different temperatures (4$55^{\circ} \mathrm{C}$ ) for $30 \mathrm{~min}$, and the relative activity was calculated by assuming that the activity 

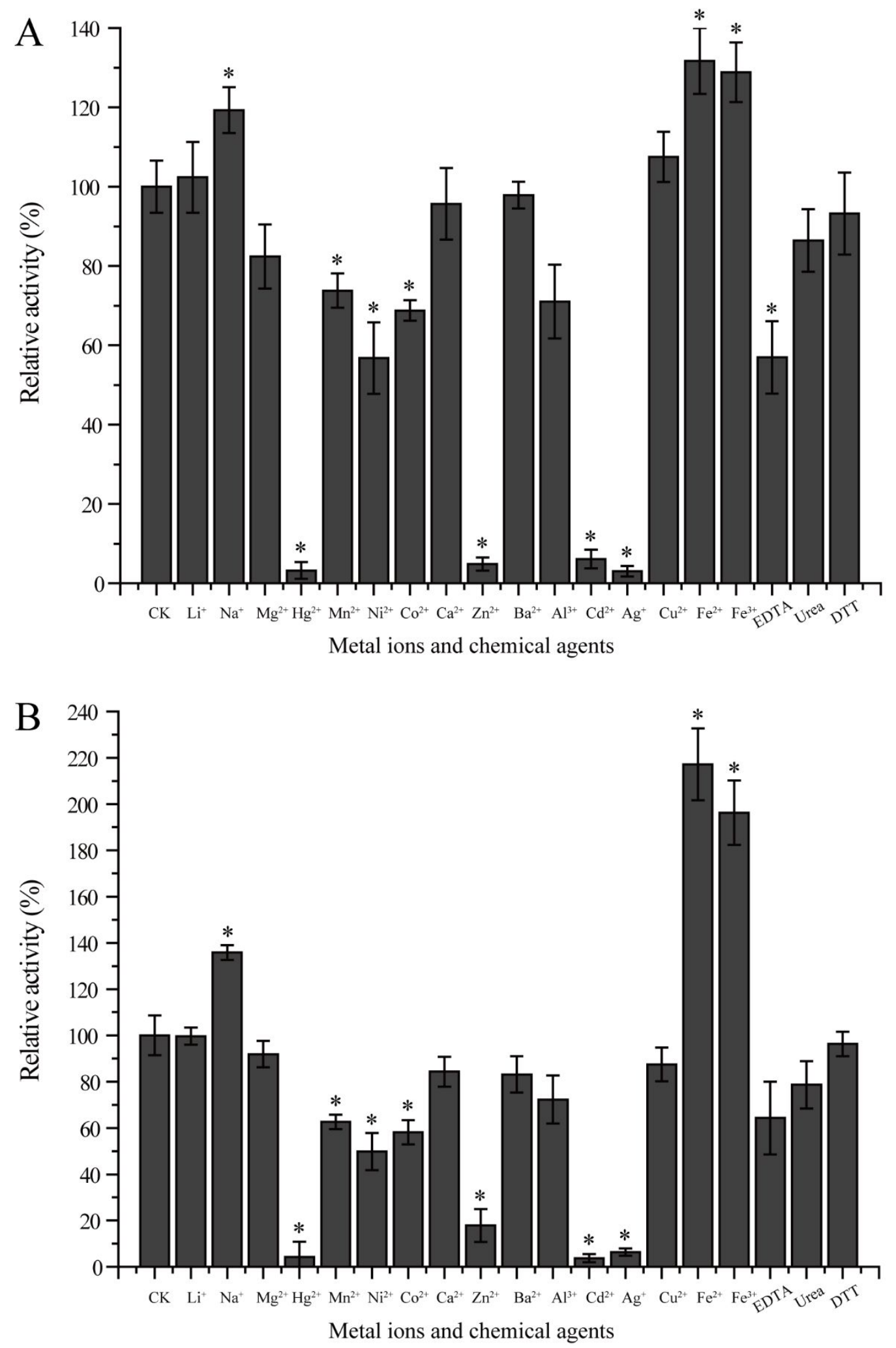

Figure S5. Effects of metal ions and chemical agents on the activities of GtdA (A) and

DsmD (B). CK: control without addition of any metal ions or chemical agents, and its 

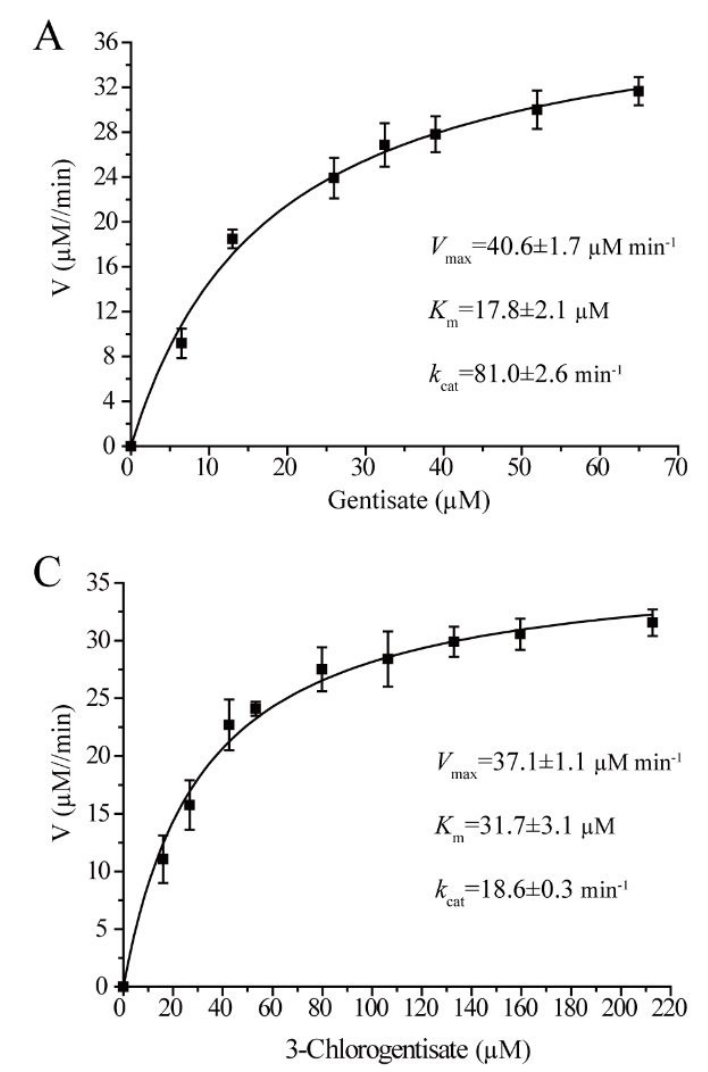
$P<0.05$ (Tukey's test).
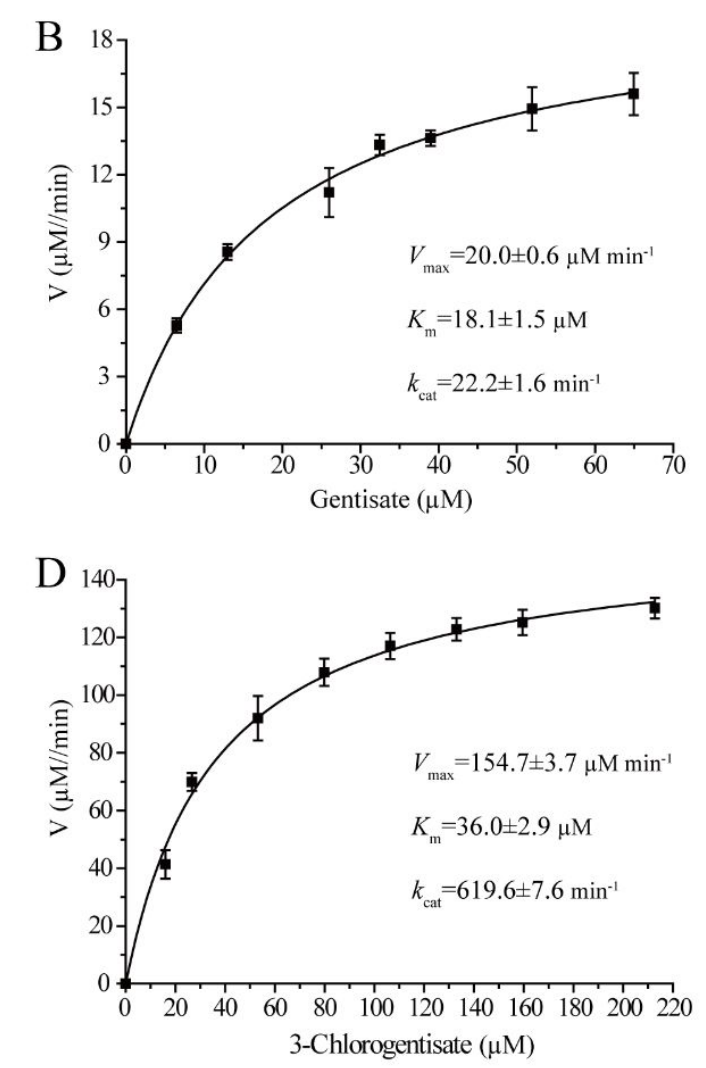

56

were $5.0 \mathrm{mM}$, and the concentration of each ion was $1.0 \mathrm{mM}$. Data are the means $\pm \mathrm{SD}$ $(n=3)$. The asterisks above the bars indicate significant difference from the control at

Figure S6. The kinetic curves of GtdA with gentisate (A) and 3-chlorogentisate (C) as substrate, and the kinetic curves of DsmD with gentisate (B) and 3-chlorogentisate (D) as substrate. Enzyme activity assays were carried out at $\mathrm{pH} 7.4$ and $30^{\circ} \mathrm{C}$ in the presence of $1.0 \mathrm{mM} \mathrm{GSH}$ and $1.0 \mathrm{mM} \mathrm{Fe}\left(\mathrm{NH}_{4}\right)_{2}\left(\mathrm{SO}_{4}\right)_{2} \cdot 6 \mathrm{H}_{2} \mathrm{O}$. The concentrations of GtdA and DsmD were $0.50 \mu \mathrm{M}$ and $0.90 \mu \mathrm{M}$, respectively, with the gentisate as substrate; the concentrations of GtdA and DsmD were $2.0 \mu \mathrm{M}$ and $0.25 \mu \mathrm{M}$, respectively, with the 3chlorogentisate as substrate. Kinetic parameters were determined by non-linear regression analysis of the initial velocity data via the Michaelis-Menten equation, 

independent experiments \pm SD.

67
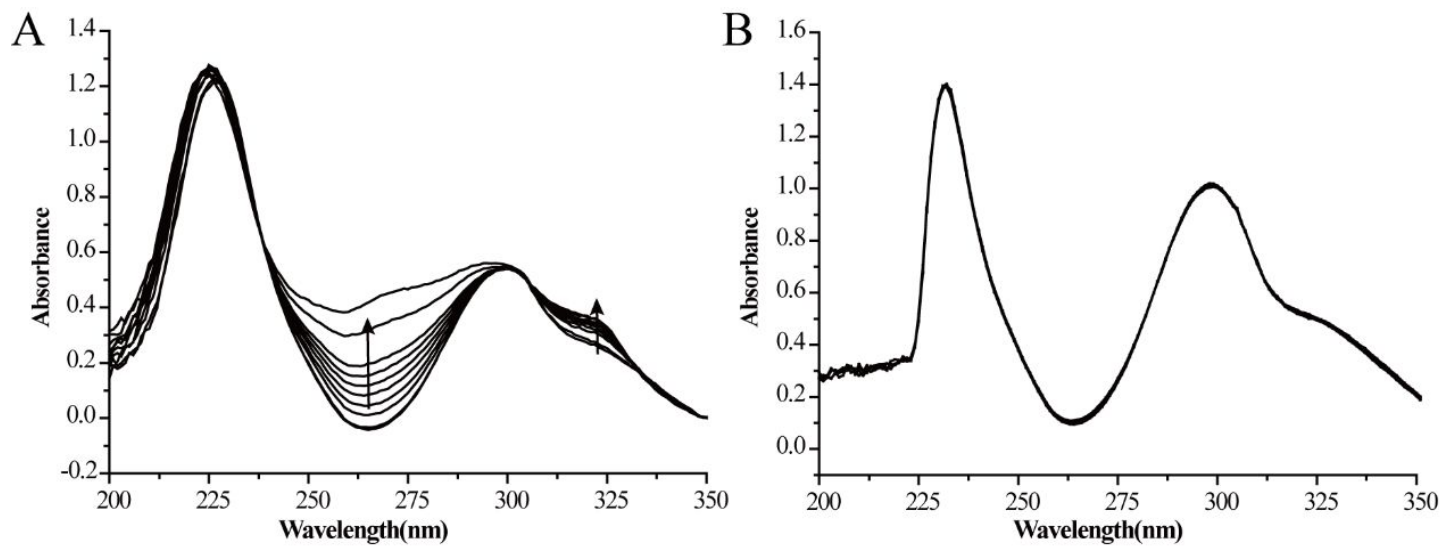

68

Figure S7. Spectrum monitoring of 3,6-DCGA in PBS buffer by UV scanning. (A) The spectra were recorded from $200 \mathrm{~nm}$ to $400 \mathrm{~nm}$ every $60 \mathrm{~s}$. The arrows indicate the direction of spectral change. 

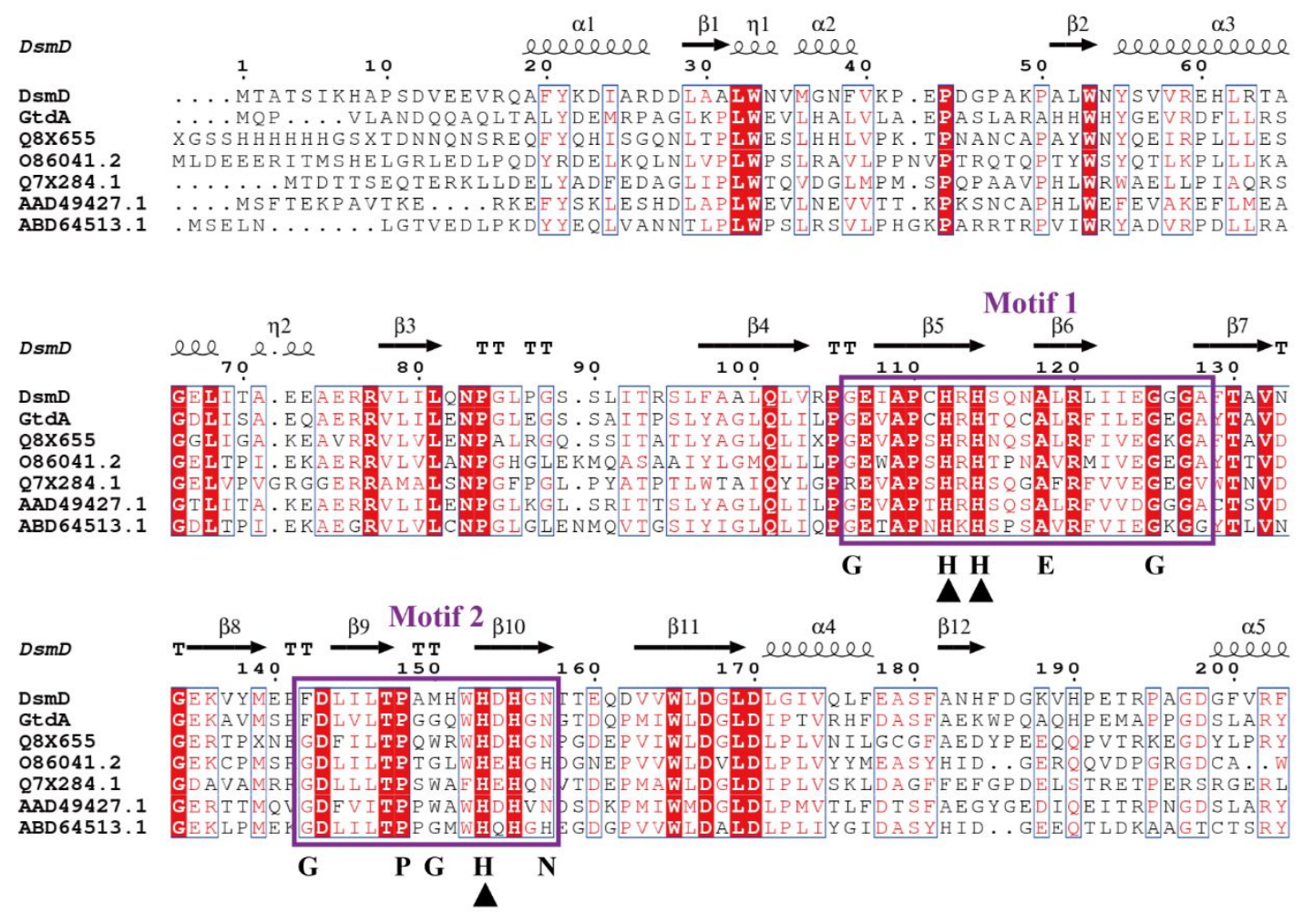

Figure S8. Alignment of the N-terminal amino acid sequences of GtdA, DsmD, and related gentisate 1,2-dioxygenases from E. coli O157:H7 (GenBank accession No: Q8X655), Ralstonia sp. U2 (O86041.2), Streptomyces sp. WA46 (Q7X284.1), Pseudomonas alcaligenes NCIB 9867 (AAD49427.1). Numbers above the amino acid sequences indicate the residues' position of DsmD. The predicted DsmD secondary structure is shown above the alignment with $\alpha$-helices, $\beta$-strands and turns. Conserved amino acids are shown in boxes, and identical amino acids are shown with red 82 background. Cupin motifs are indicated by thick boxes. Histidine residues are indicated 83 by 